\title{
Special Numbers on Analytic Functions
}

\author{
Yilmaz Simsek \\ Department of Mathematics, Faculty of Science, University of Akdeniz, Antalya, Turkey \\ Email:ysimsek@akdeniz.edu.tr
}

Received 30 January 2014; revised 28 February 2014; accepted 9 March 2014

Copyright (C) 2014 by author and Scientific Research Publishing Inc.

This work is licensed under the Creative Commons Attribution International License (CC BY).

http://creativecommons.org/licenses/by/4.0/

(c) (i) Open Access

\section{Abstract}

The aim of this paper is to give some analytic functions which are related to the generating functions for the central factorial numbers. By using these functions and $p$-adic Volkenborn integral, we derive many new identities associated with the Bernoulli and Euler numbers, the central factorial numbers and the Stirling numbers. We also give some remarks and comments on these analytic functions, which are related to the generating functions for the special numbers.

\section{Keywords}

Bernoulli Numbers, Euler Numbers, The Central Factorial Numbers, Array Polynomials, Stirling Numbers of the First Kind and the Second Kind, Generating Function, Functional Equation, Analytic Functions

\section{Introduction, Definitions and Preliminaries}

Throughout this paper, we use the following standard notations:

$\mathbb{N}=\{1,2,3, \cdots\}, \mathbb{N}_{0}=\{0,1,2,3, \cdots\}=\mathbb{N} \bigcup\{0\}$ and $\mathbb{Z}^{-}=\{-1,-2,-3, \cdots\}$. Here, $\mathbb{Z}$ denotes the set of integers, $\mathbb{R}$ denotes the set of real numbers and $\mathbb{C}$ denotes the set of complex numbers. We assume that $\ln (z)$ denotes the principal branch of the multi-valued function $\ln (z)$ with the imaginary part $\mathfrak{I}(\ln (z))$ constrained by $-\pi<\mathfrak{I}(\ln (z)) \leq \pi$. Furthermore, $0^{n}=1$ if $n=0$, and, $0^{n}=0$ if $n \in \mathbb{N}$.

$$
\left(\begin{array}{l}
x \\
v
\end{array}\right)=\frac{x(x-1) \cdots(x-v+1)}{v !}
$$

(cf. [1]-[19]).

The generating functions have been used in many branches of Mathematics and Mathematical Physics. By using $p$-adic Volkenborn integral, analytic functions and these generating functions, we derive many functional equations and differential equations. By using these equations, we derive many new identities and combinatorics relations related to these numbers and polynomials. 
The Stirling numbers of the first and the second are used in pure and applied Mathematics. The Stirling number of the second kind, denoted by $S(n, k)$, is the number of ways to partition a set of $n$ objects into $k$ groups. These numbers occur in combinatorics and in the theory of partitions. The Stirling numbers of the second kind $S(n, v)$ are defined by means of the following generating function:

$$
F_{S}(t, v)=\frac{\left(\mathrm{e}^{t}-1\right)^{v}}{v !}=\sum_{n=0}^{\infty} S(n, v) \frac{t^{n}}{n !},
$$

(cf. [1]-[19]). These numbers are computed by the following formula:

$$
x^{n}=\sum_{v=0}^{n}\left(\begin{array}{l}
x \\
v
\end{array}\right) v ! S(n, v)
$$

or

$$
S(n, v)=\frac{1}{v !} \sum_{j=0}^{v}\left(\begin{array}{l}
v \\
j
\end{array}\right)(-1)^{j}(v-j)^{n} .
$$

From the above equation, one has

$$
S(n, k)=S(n-1, k-1)+k S(n-1, k)
$$

with

$$
S(n, 0)=0(n \in \mathbb{N}) ; S(n, n)=1(n \in \mathbb{N}) ; S(n, 1)=1(n \in \mathbb{N})
$$

and $S(n, k)=0 \quad(n<k$ or $k<0)(c f .[1]-[19])$.

The Stirling numbers of the first kind $s(n, k)$ are defined by means of the following generating function:

$$
\frac{(\log (1+x))^{v}}{v !}=\sum_{n=0}^{\infty} s(n, v) \frac{t^{n}}{n !}
$$

(cf. [19]). These numbers are computed by the following formula:

$$
x(x-1) \cdots(x-n+1)=\sum_{v=0}^{n} s(n, v) x^{v} .
$$

From the above equation, one has

$$
s(n, k)=s(n-1, k-1)-(n-1) s(n-1, k),
$$

with

$$
s(n, 0)=0(n \in \mathbb{N}) ; s(n, n)=1\left(n \in \mathbb{N}_{0}\right) ; s(n, 1)=(-1)^{n-1}(n-1) !(n \in \mathbb{N})
$$

and $s(n, k)=0 \quad(n<k$ or $k<0)(c f .[19])$.

The array polynomials $\mathcal{S}_{v}^{n}(x)$ are defined by means of the following generating function:

$$
\frac{\left(\mathrm{e}^{t}-1\right)^{v}}{v !} \mathrm{e}^{t x}=\sum_{n=0}^{\infty} S_{v}^{n}(x) \frac{t^{n}}{n !},
$$

(cf. [1] [13]).

From the above generating function, one has

$$
S_{v}^{n}(x)=\frac{1}{v !} \sum_{j=0}^{v}(-1)^{v-j}\left(\begin{array}{l}
v \\
j
\end{array}\right)(x+j)^{n}
$$

with

$$
S_{0}^{0}(x)=S_{n}^{n}(x)=1, S_{0}^{n}(x)=x^{n}
$$

and for $v>n, S_{v}^{n}(x)=0 \quad(c f .[1][13])$. 


\section{Identities and Relations on the Central Factorial Numbers $T(n, k)$}

In this section, we study on generating functions for the central factorial numbers $T(n, k)$ on analytic functions. By using these functions, we derive are some functional equations. Applying these functions and equations, we give some identities and relations related to the central factorial numbers $T(n, k)$, which are defined by means of the following generating function (cf. [3] [19]):

$$
F_{T}(t, k)=\frac{1}{(2 k) !}\left(\mathrm{e}^{t}+\mathrm{e}^{-t}-2\right)^{k}=\sum_{n=k}^{\infty} T(n, k) \frac{t^{2 n}}{(2 n) !} .
$$

Srivastava and Liu [19] gave many properties and applications of the central factorial numbers.

In the work of Srivastava and Liu [19], one has the following relations:

$$
x^{n}=\sum_{k=0}^{n} T(n, k) x(x-1)\left(x-2^{2}\right)\left(x-3^{2}\right) \cdots\left(x-(k-1)^{2}\right) .
$$

Combining the above equation with (1), we also have

$$
T(n, k)=T(n-1, k-1)+k^{2} T(n-1, k),
$$

where $n \geq 1, k \geq 1,(n, k) \neq(1,1)$. For $n, k \in \mathbb{N}, T(0, k)=T(n, 0)=0$ and $T(n, 1)=1$ (cf. [19]).

In [2], Cigler gave the following formula for the numbers $T(n, k)$ :

$$
T(n, k)=\frac{1}{(2 k) !} \sum_{j=0}^{2 k}(-1)^{j}\left(\begin{array}{c}
2 k \\
j
\end{array}\right)(j-k)^{2 n} .
$$

Some properties of the function $F_{T}(t, k)$ are given as follows:

$$
F_{T}(0, k)=1 \text {. }
$$

From this property, one has $T(0,0)=1$. The function $F_{T}(t, k)$ is related to the hyperbolic functions and generating functions for the special numbers. Thus we get the following functional equation:

$$
F_{T}(t, k)=\frac{2^{k}}{(2 k) !}(\cosh (t)-1)^{k}
$$

The function $F_{T}(t, k)$ is related to the generating function for the Stirling numbers of the second kind, $S(n, k)$. That is

$$
F_{T}(t, k)=F_{S}(t, 2 k) \mathrm{e}^{-k t}
$$

where

$$
F_{S}(t, 2 k)=\frac{1}{(2 k) !}\left(\mathrm{e}^{t}-1\right)^{2 k}=\sum_{n=0}^{\infty} S(n, 2 k) \frac{t^{n}}{n !}
$$

and

$$
F_{T}(t, k)=\frac{k !}{(2 k) !} \sum_{j=0}^{k} F_{S}(t, j) F_{S}(-t, k-j) .
$$

We are going to differentiate (1) with respect to $t$ to derive a recurrence relation or identities for the central factorial numbers.

$$
\frac{\mathrm{d}}{\mathrm{d} t} F_{T}(t, k)=\frac{k(2 k-2) !}{(2 k) !}\left(\mathrm{e}^{t}-\mathrm{e}^{-t}\right) F_{T}(t, k-1)
$$

or

$$
\frac{\mathrm{d}}{\mathrm{d} t} F_{T}(t, k)=\frac{2 k(2 k-2) !}{(2 k) !} F_{T}(t, k-1) \sinh (t)
$$

Lemma 1 ([9], Lemma 11, Equation (7)] 


$$
\sum_{n=0}^{\infty} \sum_{k=0}^{\infty} A(n, k)=\sum_{n=0}^{\infty} \sum_{k=0}^{\left[\frac{n}{2}\right]} A(n, n-2 k),
$$

where $[x]$ denotes the greatest integer function.

Remark 1 Lemma 1 was generalized by Srivastava et al. [16]. By using these generalization, one may generalize Theorem 1.

Theorem 1

$$
S(n, 2 k)=n ! \sum_{j=0}^{\left[\frac{n}{2}\right]} \frac{k^{n-2 j}}{(2 j) !(n-2 j) !} T(j, k) .
$$

Proof. By combining (2) with (1), we get

$$
\sum_{n=0}^{\infty} S(n, 2 k) \frac{t^{n}}{n !}=\sum_{n=0}^{\infty} \frac{(k t)^{n}}{n !} \sum_{n=0}^{\infty} T(n, k) \frac{t^{2 n}}{(2 n) !} .
$$

By using Lemma 1, we obtain

$$
\sum_{n=0}^{\infty} S(n, 2 k) \frac{t^{n}}{n !}=\sum_{n=0}^{\infty}\left(\sum_{j=0}^{\left[\frac{n}{2}\right]} \frac{k^{n-2 j}}{(2 j) !(n-2 j) !} T(j, k)\right) t^{n} .
$$

Comparing the coefficients of $t^{n}$ on both sides of the above equation, we arrive at the desired result.

\section{Theorem 2}

$$
\sum_{j=0}^{\left[\frac{n}{2}\right]} \frac{k^{n-2 j}}{(2 j) !(n-2 j) !} T(j, k)=\frac{k !}{n !(2 k) !} \sum_{j=0}^{k} \sum_{l=0}^{n}(-1)^{k-j}\left(\begin{array}{l}
n \\
l
\end{array}\right) S_{j}^{l}(j) S(n-l, k-j),
$$

where $S_{j}^{l}(j)$ denotes the array polynomials.

Proof. By using (3), we derive the following functional equation:

$$
F_{T}(t, k) \mathrm{e}^{t x}=\frac{k !}{(2 k) !} \sum_{j=0}^{k}(-1)^{k-j} F_{A}(t, j) F_{S}(t, k-j),
$$

where $F_{A}(t, j)$ denotes generating function for the array polynomials $S_{j}^{n}(x)$. By combining (2.6) with (2.1), we obtain

$$
\frac{k !}{(2 k) !} \sum_{j=0}^{k}(-1)^{k-j} \sum_{n=0}^{\infty} S_{j}^{n}(j) \frac{t^{n}}{n !} \sum_{n=0}^{\infty} S(n, k-j) \frac{t^{n}}{n !}=\sum_{n=0}^{\infty} \frac{(k t)^{n}}{n !} \sum_{n=0}^{\infty} T(n, k) \frac{t^{2 n}}{(2 n) !} .
$$

By using Lemma 1, we obtain

$$
\sum_{n=0}^{\infty}\left(\sum_{j=0}^{\left[\frac{n}{2}\right]} \frac{k^{n-2 j}}{(2 j) !(n-2 j) !} T(j, k)\right) t^{n}=\sum_{n=0}^{\infty}\left(\frac{k !}{n !(2 k) !} \sum_{j=0}^{k} \sum_{l=0}^{n}(-1)^{k-j}\left(\begin{array}{l}
n \\
l
\end{array}\right) S_{j}^{l}(j) S(n-l, k-j)\right) \frac{t^{n}}{n !} .
$$

Comparing the coefficients of $t^{n}$ on both sides of the above equation, we arrive at the desired result.

Remark 2 By using functional equation in (2.3), (2.4) and (2.5), one may also obtain an interesting identities and relationships between the Stirling numbers of the second kind and the central factorial numbers.

\section{Identities Related to the $p$-Adic Volkenborn Integral and the Central Factorial Numbers}

In this section, we give applications of the $p$-adic integral on $\mathbb{Z}_{p}$, set of $p$-adic integers. By using the Witt's formula for the Bernoulli and Euler numbers related to the $p$-adic Volkenborn integral and the Mahler coefficients, we derive many interesting and novel identities associated with the central factorial numbers, the family of num- 
bers $C_{n}^{(x)}$, the Stirling numbers of the first and the second kind and also combinatorics identities.

We use notations of the work of Kim [4] for the $p$-adic $q$-Volkenborn integral. Let $p$ be a fixed prime. It is known that

$$
\mu_{q}\left(x+p^{N} \mathbb{Z}_{p}\right)=\frac{q^{x}}{\left[p^{N}\right]_{q}}
$$

is a distribution on $\mathbb{Z}_{p}$ for $q \in \mathbb{C}_{p}$ with $|1-q|_{p}<1$, cf. [4]. Let $U D\left(\mathbb{Z}_{p}\right)$ be the set of uniformly differentiable functions on $\mathbb{Z}_{p}$. The $p$-adic $q$-integral of the function $f \in U D\left(\mathbb{Z}_{p}\right)$ is defined by Kim [4] as follows:

$$
\int_{\mathbb{Z}_{p}} f(x) \mathrm{d} \mu_{q}(x)=\lim _{N \rightarrow \infty} \frac{1}{\left[p^{N}\right]_{q}} \sum_{x=0}^{p^{N}-1} f(x) q^{x},
$$

where

$$
[x]=\frac{1-q^{x}}{1-q} .
$$

The bosonic $p$-adic integral ( $p$-adic Volkenborn integral) was considered from a physical point of view to the bosonic limit $q \rightarrow 1$, as follows ([4]):

$$
\int_{\mathbb{Z}_{p}} f(x) \mathrm{d} \mu_{1}(x)=\lim _{N \rightarrow \infty} \frac{1}{p^{N}} \sum_{x=0}^{p^{N}-1} f(x),
$$

where

$$
\mu_{1}\left(x+p^{N} \mathbb{Z}_{p}\right)=\frac{1}{p^{N}}
$$

The $p$-adic $q$-integral is used in many branch of mathematics, mathematical physics and other areas (cf. [4] [11] [14] [18]).

By using (7), we have the Witt's formula for the Bernoulli numbers $B_{n}$ as follows:

$$
\int_{\mathbb{Z}_{p}} x^{n} \mathrm{~d} \mu_{1}(x)=B_{n}
$$

(cf. [4] [5] [11] [14] [18]).

We consider the fermionic integral in contrast to the bosonic integral, which is called the fermionic $p$-adic Volkenborn integral on $\mathbb{Z}_{p}$ cf. [5]. That is

$$
\int_{\mathbb{Z}_{p}} f(x) \mathrm{d} \mu_{-1}(x)=\lim _{N \rightarrow \infty} \sum_{x=0}^{p^{N}-1}(-1)^{x} f(x)
$$

where

$$
\mu_{1}\left(x+p^{N} \mathbb{Z}_{p}\right)=\frac{(-1)^{x}}{p^{N}}
$$

(cf. [5]). By using (9), we have the Witt's formula for the Euler numbers $E_{n}$ as follows:

$$
\int_{\mathbb{Z}_{p}} x^{n} \mathrm{~d} \mu_{-1}(x)=E_{n},
$$

(cf. [5] [6] [11] [18]).

\section{Theorem 3}

$$
\int_{\mathbb{Z}_{p}}\left(\begin{array}{l}
x \\
j
\end{array}\right) \mathrm{d} \mu_{1}(x)=\frac{(-1)^{j}}{j+1} .
$$


Proof of Theorem 3 was given by Schikhof [14].

Theorem 4

$$
\int_{\mathbb{Z}_{p}}\left(\begin{array}{c}
x \\
j
\end{array}\right) \mathrm{d} \mu_{-1}(x)=\frac{(-1)^{j}}{2^{j}} .
$$

Proof of Theorem 4 was given by Kim et al. ([7] [8]).

The family of numbers $C_{n}^{(x)}$ was defined by Srivastava and Liu [19]. These numbers are related to the Stirling numbers of the first and the second kind, which are given by the following theorem.

Theorem 5 ([19], Theorem 1]) Let $n \geq k \quad(n, k \in \mathbb{N})$ and

$$
w(n, k)=(-1)^{k} \sum_{j=k}^{n} 2^{n-j} S(n, j) s(j, k) .
$$

Then

$$
C_{n}^{(x)}=\sum_{k=1}^{n} w(n, k) x^{k}
$$

In [19], Equation-(2.3)], Srivastava and Liu also gave the following relation:

$$
C_{n}^{(x)}=\sum_{j=0}^{n}(-1)^{j} j ! 2^{n-j}\left(\begin{array}{c}
x+j-1 \\
j
\end{array}\right) S(n, j) .
$$

By using the above formulae, we derive the following identities related to the numbers $C_{n}^{(x)}$, the Stirling numbers, the Bernoulli numbers and the Euler numbers.

According to the work of Kim at al. ([7] [8]), we get

$$
\int_{\mathbb{Z}_{p}}\left(\begin{array}{c}
-x \\
j
\end{array}\right) \mathrm{d} \mu_{-1}(x)=\int_{\mathbb{Z}_{p}}\left(\begin{array}{c}
x+j-1 \\
j
\end{array}\right) \mathrm{d} \mu_{-1}(x) .
$$

Combining Theorem 4 with the above equation, we get

$$
\int_{\mathbb{Z}_{p}}\left(\begin{array}{c}
x+n-1 \\
n
\end{array}\right) \mathrm{d} \mu_{-1}(x)=\sum_{m=0}^{n}(-1)^{m}\left(\begin{array}{c}
n-1 \\
n-m
\end{array}\right) 2^{-m} .
$$

Integrating both sides of (3.7) with respect to the fermionic integral on $\mathbb{Z}_{p}$, and than using (3.8), we obtain the following Lemma:

\section{Lemma 2}

$$
\int_{\mathbb{Z}_{p}} C_{n}^{(x)} \mathrm{d} \mu_{-1}(x)=\sum_{j=0}^{n}(-1)^{j} j ! S(n, j) \sum_{m=0}^{j}(-1)^{m}\left(\begin{array}{c}
j-1 \\
j-m
\end{array}\right) 2^{n-m-j} .
$$

Integrating both sides of (3.6) with respect to the fermionic integral on $\mathbb{Z}_{p}$, and than using (3.4), we obtain the following Lemma:

\section{Lemma 3}

$$
\int_{\mathbb{Z}_{p}} C_{n}^{(x)} \mathrm{d} \mu_{-1}(x)=\sum_{k=0}^{n} E_{k} w(n, k),
$$

where $E_{k}$ denotes the Euler numbers.

By using Lemma 2 and Lemma 3, we arrive at the following theorem:

\section{Theorem 6}

$$
\sum_{k=0}^{n} E_{k} w(n, k)=\sum_{j=0}^{n}(-1)^{j} j ! S(n, j) \sum_{m=0}^{j}(-1)^{m}\left(\begin{array}{c}
j-1 \\
j-m
\end{array}\right) 2^{n-m-j} .
$$


Similarly, according to the work of Kim et al. ([7] [8]), we get

$$
(-1)^{n} \int_{\mathbb{Z}_{p}}\left(\begin{array}{c}
-x \\
j
\end{array}\right) \mathrm{d} \mu_{1}(x)=\int_{\mathbb{Z}_{p}}\left(\begin{array}{c}
x+n-1 \\
n
\end{array}\right) \mathrm{d} \mu_{1}(x) .
$$

Combining Theorem 3 with the above equation, we get

$$
\int_{\mathbb{Z}_{p}}\left(\begin{array}{c}
x+j-1 \\
j
\end{array}\right) \mathrm{d} \mu_{1}(x)=\sum_{m=0}^{j}(-1)^{m}\left(\begin{array}{c}
j-1 \\
j-m
\end{array}\right) \frac{1}{m+1} .
$$

Integrating both sides of (3.7) with respect to the bosonic $p$-adic integral on $\mathbb{Z}_{p}$, and than using (3.9), we obtain the following lemma:

\section{Lemma 4}

$$
\int_{\mathbb{Z}_{p}} C_{n}^{(x)} \mathrm{d} \mu_{1}(x)=\sum_{j=0}^{n}(-1)^{j} j ! S(n, j) \sum_{m=0}^{j}(-1)^{m}\left(\begin{array}{c}
j-1 \\
j-m
\end{array}\right) \frac{2^{n-j}}{m+1} .
$$

Integrating both sides of (3.6) with respect to the fermionic integral on $\mathbb{Z}_{p}$, and than using (3.2), we obtain the following lemma:

\section{Lemma 5}

$$
\int_{\mathbb{Z}_{p}} C_{n}^{(x)} \mathrm{d} \mu_{1}(x)=\sum_{k=0}^{n} B_{k} w(n, k),
$$

where $B_{k}$ denotes the Bernoulli numbers.

By using Lemma 4 and Lemma 5, we arrive at the following theorem:

\section{Theorem 7}

$$
\sum_{k=0}^{n} B_{k} w(n, k)=\sum_{j=0}^{n}(-1)^{j} j ! S(n, j) \sum_{m=0}^{j}(-1)^{m}\left(\begin{array}{c}
j-1 \\
j-m
\end{array}\right) \frac{2^{n-j}}{m+1} .
$$

\section{Acknowledgements}

The paper was supported by the Scientific Research Project Administration of Akdeniz University.

\section{References}

[1] Chang, C.-H. and Ha, C.-W. (2006) A Multiplication Theorem for the Lerch Zeta Function and Explicit Representations of the Bernoulli and Euler polynomials. Journal of Mathematical Analysis and Applications, 315, 758-767. http://dx.doi.org/10.1016/j.jmaa.2005.08.013

[2] Cigler, J. Fibonacci Polynomials and Central Factorial Numbers. Preprint. http://homepage.univie.ac.at/johann.cigler/preprints/central-factorial.pdf

[3] Comtet, L. (1974) Advanced Combinatorics: The Art of Finite and Infinite Expansions. Reidel, Dordrecht and Boston, (Translated from the French by J. W. Nienhuys).

[4] Kim, T. (2002) q-Volkenborn integration. Russian Journal of Mathematical Physics, 19, 288-299.

[5] Kim, T. (2006) q-Euler Numbers and Polynomials Associated with p-Adic $q$-Integral and Basic q-zeta Function. Trends in International Mathematics and Science Study, 9, 7-12.

[6] Jang, L.C. and Kim, T. (2008) A New Approach to q-Euler Numbers and Polynomials. Journal of Concrete and Applicable Mathematics, 6, 159-168.

[7] Kim, D.S. and Kim, T. (2013) Daehee Numbers and Polynomials. Applied Mathematical Sciences, 7, 5969-5976.

[8] Kim, D.S., Kim, T. and Seo, J. (2013) A Note on Changhee Numbers and Polynomials. Advanced Studies in Theoretical Physics, 7, 993-1003.

[9] Rainville, E.D. (1960) Special Functions. The Macmillan Company, New York.

[10] Simsek, Y. (2010) On q-Deformed Stirling numbers. International Journal of Computer Mathematics, 15, 70-80.

[11] Simsek, Y. (2010) Complete sum of products of $(h, q)$-Extension of Euler Polynomials and Numbers. Journal of Difference Equations and Applications, 16, 1331-1348. http://dx.doi.org/10.1080/10236190902813967 
[12] Simsek, Y. (2013) Identities Associated with Generalized Stirling Type Numbers and Eulerian Type Polynomials. Mathematical and Computational Applications, 18, 251-263.

[13] Simsek, Y. (2013) Generating Functions for Generalized Stirling type Numbers, Array Type Polynomials, Eulerian Type Polynomials and Their Applications. Fixed Point Theory and Applications, 87, 343-1355.

[14] Schikhof, W.H. (1984) Ultrametric Calculus: An Introduction to p-Adic Analysis. Cambridge Studies in Advanced Mathematics 4, Cambridge University Press, Cambridge.

[15] Srivastava, H.M. (2011) Some Generalizations and Basic (or q-) Extensions of the Bernoulli, Euler and Genocchi Polynomials. Applied Mathematics \& Information Sciences, 5, 390-444.

[16] Srivastava, H.M., Ozarslan, M.A. and Kaanoğlu, C. (2010) Some Families of Generating Functions for a Certain Class of Three-Variable Polynomials. Integral Transforms and Special Functions, 21, 885-896. http://dx.doi.org/10.1080/10652469.2010.481439

[17] Srivastava, H.M. and Choi, J. (2012) Zeta and q-Zeta Functions and Associated Series and Integrals. Elsevier Science Publishers, Amsterdam, London and New York.

[18] Srivastava, H.M., Kim, T. and Simsek, Y. (2005) q-Bernoulli Numbers and Polynomials Associated with Multiple q-Zeta Functions and Basic L-Series. Russian Journal of Mathematical Physics, 12, 241-268.

[19] Srivastava, H.M. and Liu, G.-D. (2009) Some Identities and Congruences Involving a Certain Family of Numbers. Russian Journal of Mathematical Physics, 16, 536-542. http://dx.doi.org/10.1134/S1061920809040086 